\title{
Modern Reverse Engineering Methods Used to Modification of Jewelry
}

\author{
Arkadiusz Kroma ${ }^{1 *}$, Oliwia Adamczak ${ }^{1}$, Robert Sika ${ }^{1}$, Filip Górski², \\ Wiesław Kuczko², Krzysztof Grześkowiak \\ 1 Division of Foundry and Plastic Working, Poznan University of Technology, ul. Piotrowo 3, 61-138 Poznan, \\ Poland \\ 2 Division of Production Engineering, Poznan University of Technology, ul. Piotrowo 3, 61-138 Poznan, Poland \\ * Corresponding author's e-mail: arkadiusz.kroma@put.poznan.pl
}

\begin{abstract}
The article presents the results of research aimed at redesigning a jewelry product with the use of modern reverse engineering methods. A detailed review of the literature was conducted and the essence of the application of this technology in the era of Industry 4.0 was presented. The 3D digitization of the research object was performed with the use of Aicon SmartSCAN-HE R8 and DAVID-SLS3 scanners. The obtained better quality file was edited in Autodesk Meshmixer 3.5 software. On the basis of the created model, a 3D print was made using FDM (Fused Deposition Modeling) and DPP (Daylight Polymer Printing) technology with two different materials - HIPS (High Impact Polystyrene) and photopolymer casting resin. The final stage of the work was the measurement of the surface roughness of the B101 (CuSn10P) tin-phosphorus alloy castings made using the method of investment casting.
\end{abstract}

Keywords: 3D scan, 3D printing, DPP, FDM, Aicon, David-sls, Reverse engineering.

\section{INTRODUCTION}

Jewelry is a dynamically developing branch. Over the years, the services provided have changed and expanded their scope. New technologies related to the design and production of jewelry have been developed [33]. Currently, the jewelry industry is characterized by a high level of quality, repeatability and precision compared to previous years.

Nowadays, people value originality, which makes the implementation of individual projects very popular. The ubiquitous computerization of manufacturing $[11,13,16]$ and design processes $[1,5,25,37]$ have largely contributed to this $[14 \div 15,22,27]$. These activities became a part of the prevailing smart \& sustainable manufacturing trends $[18,36]$. One of the modern computer design processes that is used increasingly often, is the process of reverse engineering, the essence of which is to enable the reconstruction of defects in the already existing products or their complete redesign according to the clients' preferences. For this purpose, scanning devices are used to digitize precision products that can be further processed. The completed project is fed to the $3 \mathrm{D}$ printer, which produces the initial model (pattern / casting model) $[2 \div 4,38]$. Owing to this, it is possible to make the first try-on parts, check the real size of the designed element and most importantly, make the final product based on it. The last stage of this process is the production of the redesigned product by means of the casting process and its final finishing, e.g. removal of visible defects, polishing or the deposition of additional elements, e.g. stones [26, 33].

Reverse engineering is the reproduction of the actual product structure as well as implementation of new ideas related to them. It is a process that brings a number of innovative solutions with it. Reverse engineering makes it possible to recreate elements based on the information 
about a physical object by analyzing and processing them [29]. It is one of the elements of the fourth industrial revolution, a system integrating industrial infrastructure, i.e. machines, with information technologies and the Internet $[9,10,30]$. This issue covers the additive technologies, such as 3D design, 3D scanning and 3D printing, which are the main stages of the process of recreating elements [35].

After generating virtual models of physical objects, it is possible to analyze them, implement individual changes to the internal and external structure, and perform strength tests of elements. The most useful application of reverse engineering to improve the manufacturing process is the production of spare parts. Owing to the development of this technology, the process has gained popularity. In the times of Industry 4.0, reverse engineering is used in various fields, i.e. [24]:

- automotive (obtaining parts of the vehicles that have long been withdrawn from production),

- aviation (obtaining spare parts for airplanes that are required after several years of use),

- $\operatorname{art}$ (recreating artistic objects, e.g. sculptures),

- medicine (manufacturing of implants) $[32,34]$,

- computer graphics (obtaining 3D data from a model or sculpture for animation in games and movies),

- jewelry (obtaining information from precision products for the production of individual projects).

The use of a process belonging to the rapid production methods (rapid prototyping) by jewelers significantly increases the flexibility of the work performed in a much shorter time than in the case of the traditional methods. The use of reverse engineering in this field allows saving time and performing complex patterns. Jewelry workshops should have modern machinery in order to perform the services based on the reverse engineering process. For the production of jewelry, appropriate 3D scanners [6], computers with special CAD software (ComputerAided Design) $[25,31]$ and 3D printers are used, which play a significant role. Such devices testify to the innovativeness of the jewelry production plant $[17,30]$.

Consumers in the 21 st century focus primarily on convenience and thus the option of purchasing in the Internet. The buyer has the option to remotely commission a jewelry company to manufacture precise products according to his requirements. It works on the basis of B2C (Business-to-Consumer) and C2B (Consumer-to-Business). Entrepreneurs want to satisfy their clients at every stage; therefore it is important to follow the trends in a given period [23].

This article deals with the application of reverse engineering to redesign a selected jewelry element in the aspect of the impact of various materials and technologies used in $3 \mathrm{D}$ printing.

\section{METHODOLOGY}

The described product was digitized using two selected 3D scanners. The first was the 3D structured light scanner - DAVID SLS-3 $[19 \div 21,28]$. The device is designed to digitize larger items. For example, it is used for scanning prostheses of body parts. The working area of the device is $60-500 \mathrm{~mm}$, and its accuracy is $0.05 \mathrm{~mm}$. The test setup consisted of an industrial camera with a high-quality lens, video projector, computer workstation and research model. Despite the purpose of the device for scanning larger objects, it was decided to test its operation with the use of a jewelry product.

The second scan was carried out using the Aicon SmartSCAN-HE R8 optical contactless scanner (Fig. 1). The device enables 3D digitization of small-sized precision products and is equipped with white light technology, enabling to read additional information about the color required at the design stage. The accuracy of the measurement is $0.01 \mathrm{~mm}$. The test setup consisted of a computer workstation with dedicated Optocat software [12] (it includes all the steps of the 3D scanning process - calibration, scanning and final data processing and evaluation), scanner, research object and a special substrate.

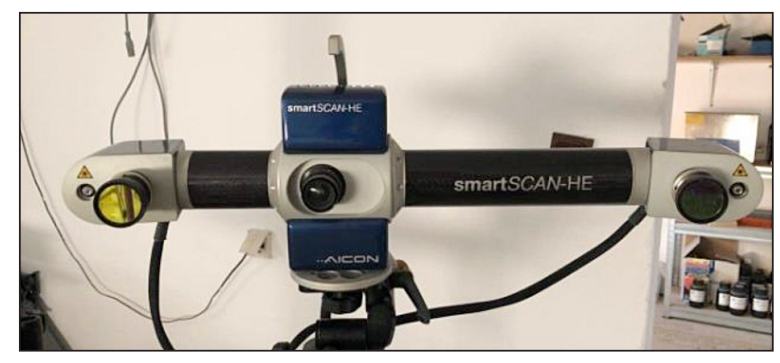

Fig. 1. Aicon SmartSCAN-HE R8 optical scanner 
At the beginning, the research object, which was a brooch, was carefully covered with an antireflective coating. The next step was to start the automatic three-dimensional scanning process. The projection unit projected a sequence of fringes onto the measurement object. The scan result was obtained in the STL format. At the end of this stage, the data related to the scanned object was processed (edited) in Optocat.

The obtained geometries were imported to Autodesk Meshmixer 3.5. It is software intended for editing 3D models, among others for 3D printing. It has the function of merging many models into one, redesigning the object and perfecting the details. The program has tools that allow carving, transforming, connecting and cutting the surfaces of the imported files, etc.

The developed models were printed using the Zortrax M200 [39] and Liquid Crystal HR [7]. The Zortrax M200 printer (Fig. 2) uses FDM technology, has a minimum layer thickness of $0.09 \mathrm{~mm}$, a working area of $200 \times 200 \times 180 \mathrm{~mm}$ and a $0.4 \mathrm{~mm}$ nozzle.

The Liquid Crystal HR printer (Fig. 3) uses DPP technology, the minimum layer thickness is $0.05 \mathrm{~mm}$, and the working area with a resolution of 97 microns is $196 \times 147 \times 250 \mathrm{~mm}$.

On the basis of the printed models, the molds needed to make the castings using the centrifugal force were prepared. First, the material of

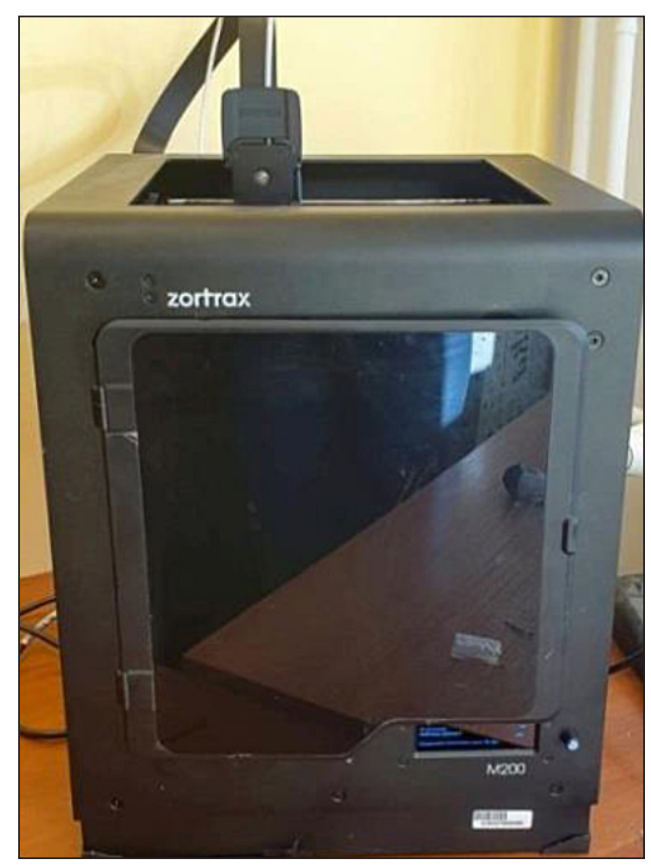

Fig. 2. Zortrax M200 printer the gating systems was melted with a soldering iron, then the models were assembled. The prepared elements (the so-called "sprue trees") were mounted in rubber washers on which the metal sleeves were mounted. In the next step, gypsum mass was prepared and poured over the molds. Finally, the molds were placed for about 5 min in a vacuum pump to deaerate the material. After two hours, the mass set and the molds were placed into the Nabertherm N 150 WAX furnace for the annealing process (Fig. 4). The mold annealing time was $12.5 \mathrm{~h}$ [8].

An appropriate amount of material $(\mathrm{CuS}-$ n10P) for casting process was prepared. The crucible with the alloy was placed in an induction furnace. The tin-phosphor alloy was heated to the flow temperature. The previously made mold was placed in the centrifugal force pouring machine (Fig. 5). The filled mold was put into the water. The casting was pulled out from the liquid. The above-mentioned casting procedure was used for all 3D printed models.

In the final stage, the roughness of the castings was measured using a MarSurf PS 10 profilometer by MAHR. The measurement consists in examining surface irregularities with a transducer (mechanical, piezo/photo electric or induction), which converts the irregularities of the test surface into an electrical signal in the form of $\mathrm{Ra}$, Rz. The test setup is shown in Figue 6.

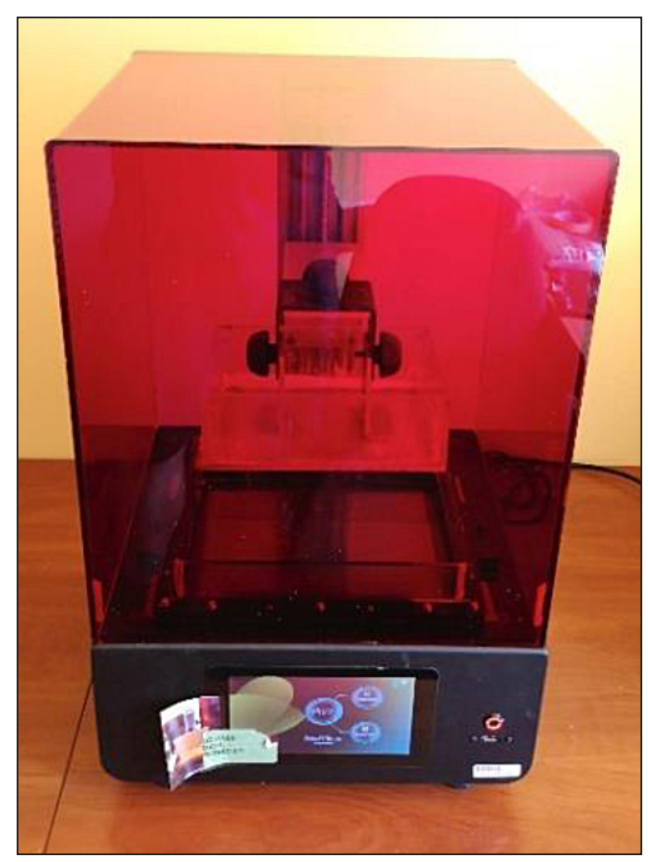

Fig. 3. Liquid Crystal HR printer 


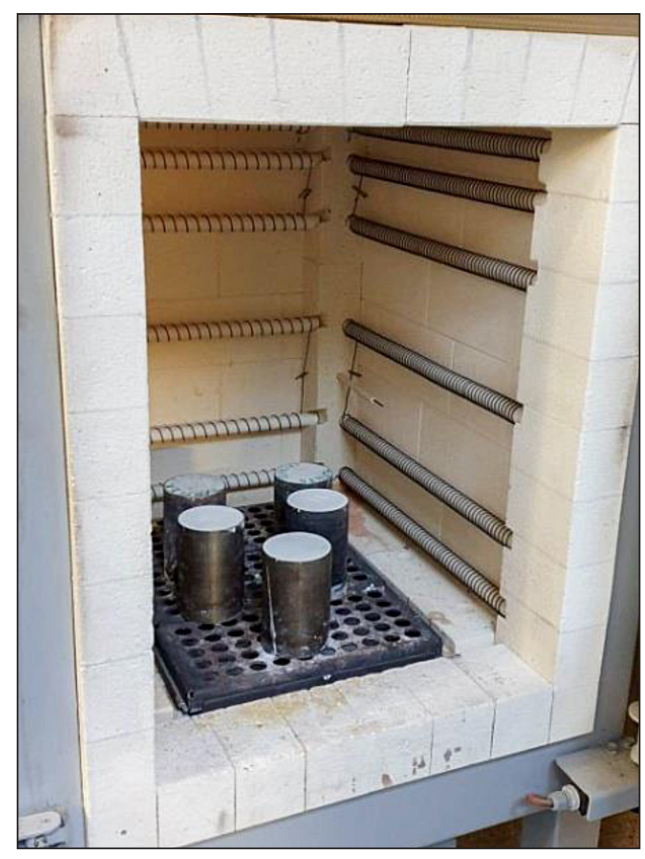

Fig. 4. Molds in the Nabertherm N 150 WAX furnace

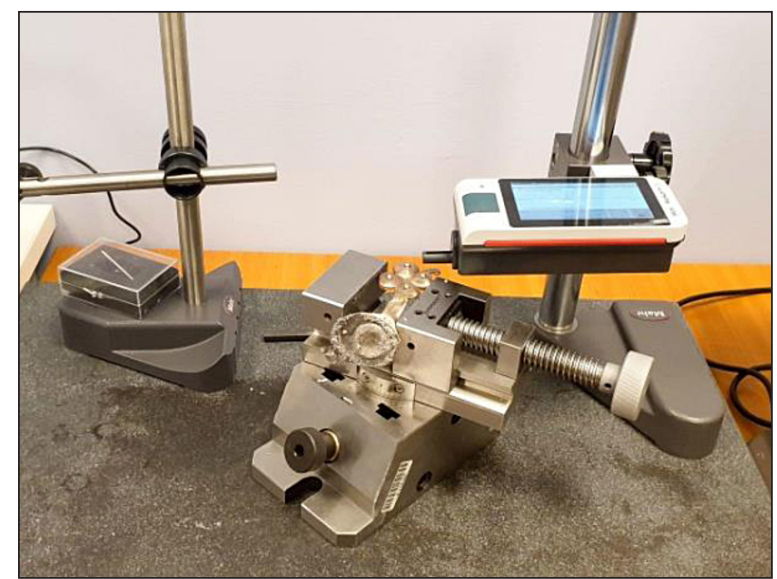

Fig. 6. Setup for testing the surface quality of the castings

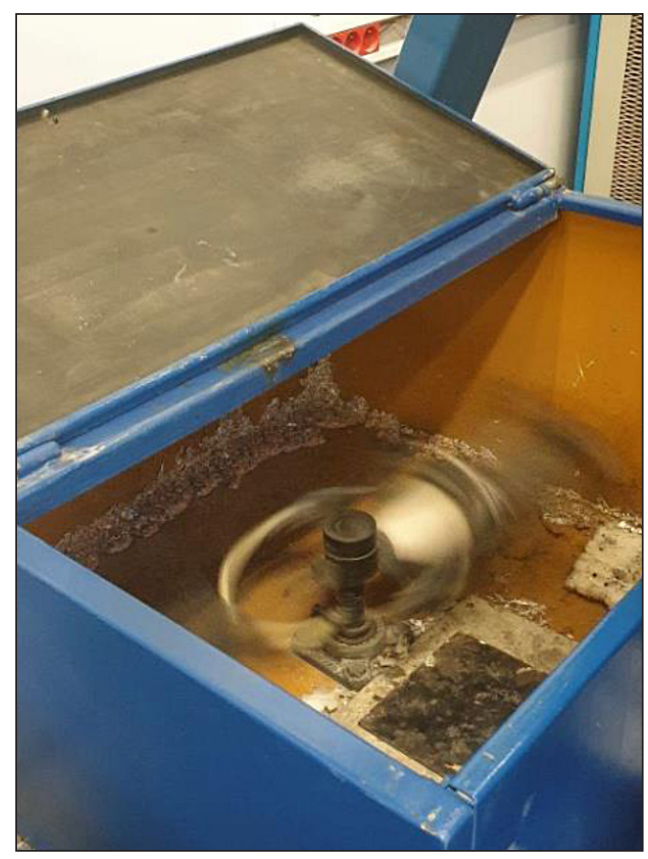

Fig. 5. Setup for casting using the centrifugal force

\section{RESULTS AND DISCUSSION}

Scanning with the DAVID SLS-3 device was not as precise as with the Aicon SmartSCANHE R8. The second scanner had a more precise camera that more accurately reproduced small objects. The DAVID SLS-3 device is not suitable for digitizing precision products. It is much better suited for scanning larger items, e.g. body parts for making limb prostheses. The resulting scans made on this device contain imprecise data on the shape of the object, which relocated into the difficulty of making the model. The scanned object and the result of the obtained models are shown in Figure 7.

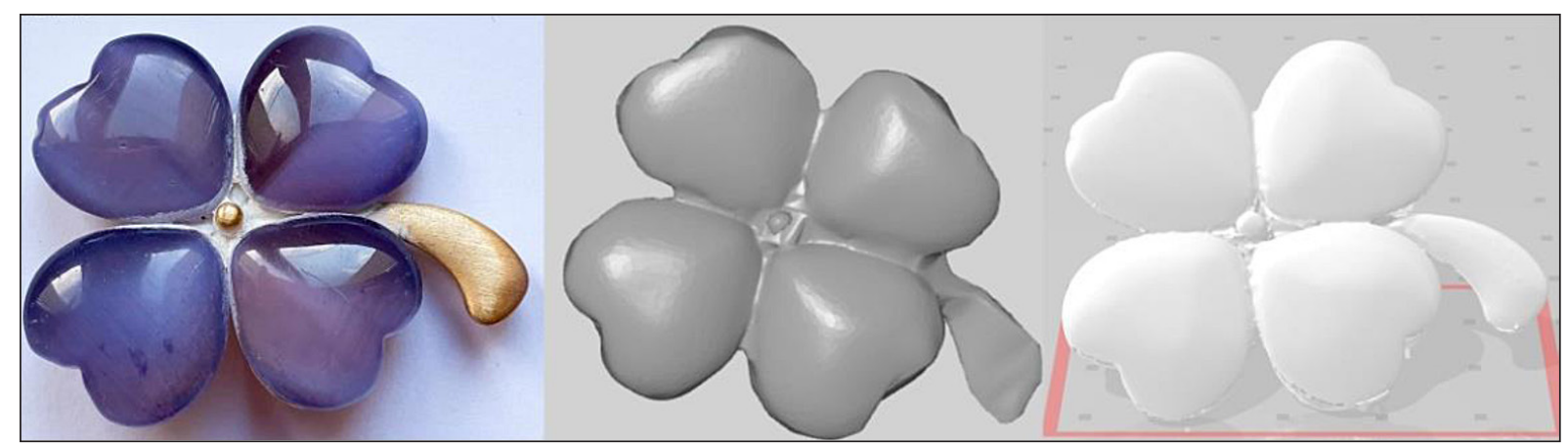

Fig. 7. The real object and the models obtained by the DAVID-SLS3 and Aicon SmartSCAN-HE R8 scanner (from the left) 
After the analysis of the surface discontinuities of the obtained files (visible defects in the structure or non-geometric edges deviating from the original with the naked eye), a decision was made to choose the model generated by the Aicon device for further editing.

At the beginning of the model processing, a triangle mesh with many defects was examined (Fig. 8). Red and blue references indicate discontinuities in the model mesh, while the magenta spheres refer to relatively small areas that are disconnected from the whole.

Then, by selecting the "Auto repair" tool, most errors were automatically removed (Fig. 9).
The remaining defects in the form of holes and cavities were filled manually with the "Smooth MVC" tool (Fig. 10).

Finally, the model was redesigned by adding hemispheres to each leaf of the model. The final effect is shown in Figure 11.

The next step was to manufacture the created project. The basic process data of $3 \mathrm{D}$ printing is presented in the Table 1 and its results are shown in Figure 12.

Then, after preparation and annealing, the molds were poured with the B101 (CuSn10P) liquid alloy using the centrifugal force, to obtain the castings shown in Figure 13.

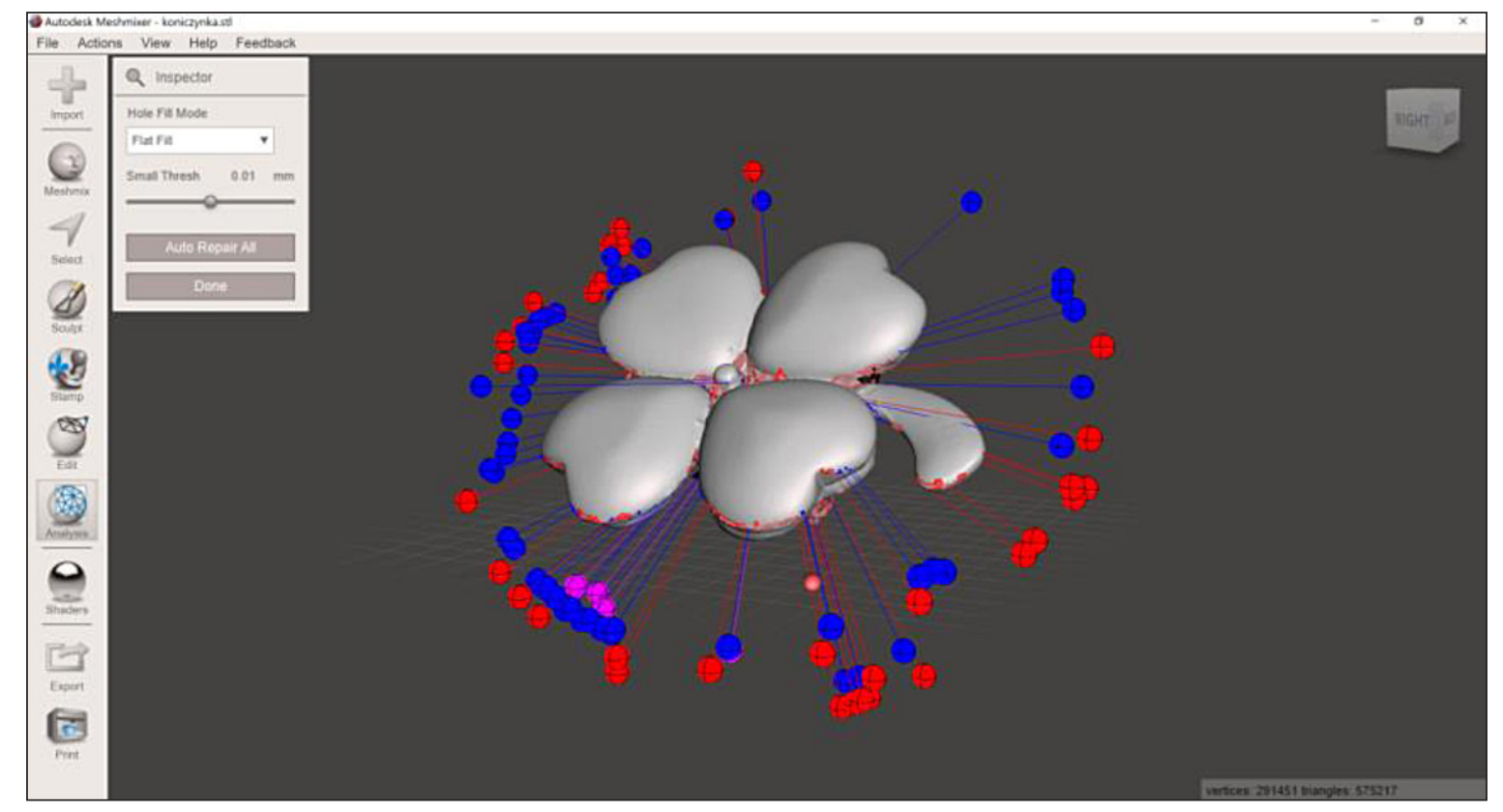

Fig. 8. Errors of the obtained mesh

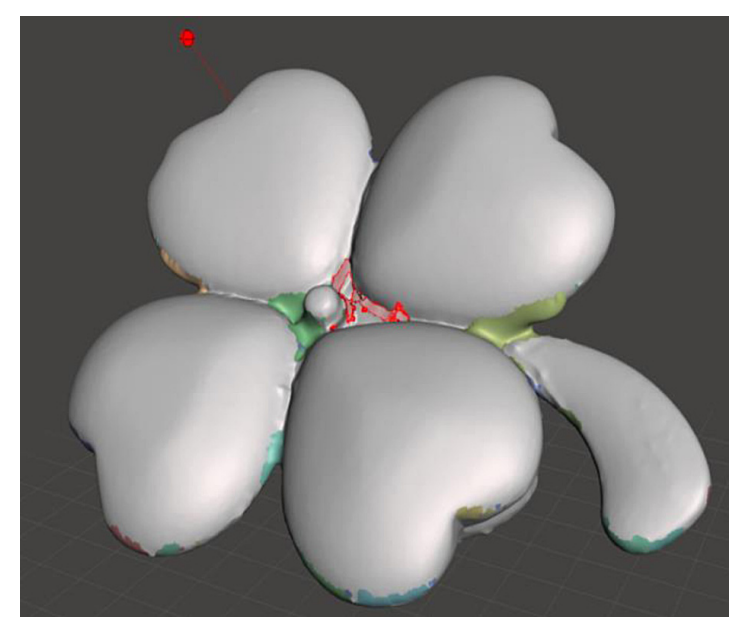

Fig. 9. Model after automatic repair

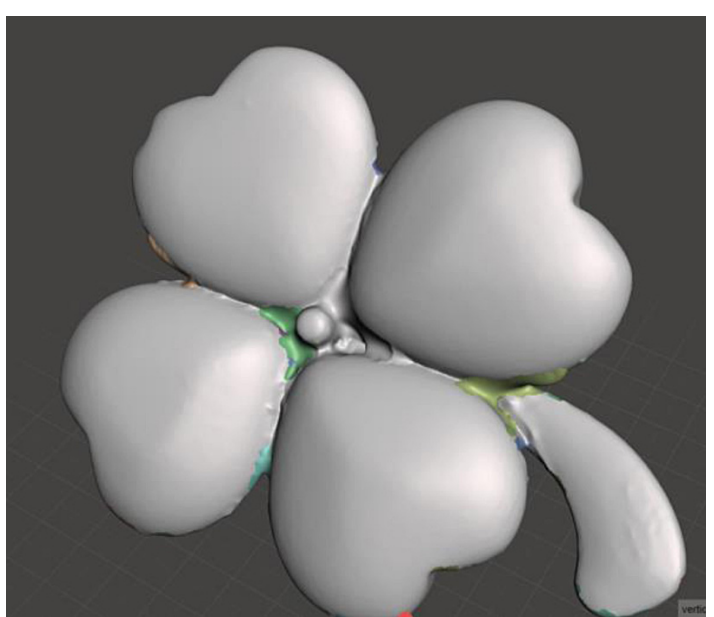

Fig. 10. The model prepared for the redesign stage 


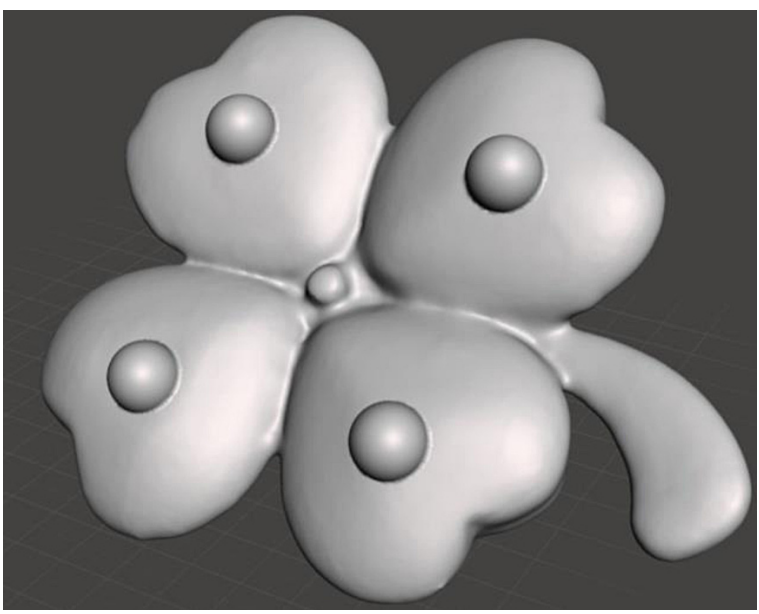

Fig. 11. Final redesigned model

In the last stage, the surface roughness was measured. Due to the large curvature of the casting surface, it was decided to use the lower, least curved part of the casting for the tests. The Ra roughness measurement results are shown in Table 2, whereas the graphs are shown in Figures 14-15. The calculated standard deviation is shown in Table 3.

On the basis of the data above, it was concluded that the better surface quality was obtained in a casting made on the basis of a resin model. Standard deviation and Ra roughness index values are smaller compared to the values obtained on the casting made of the HIPS.

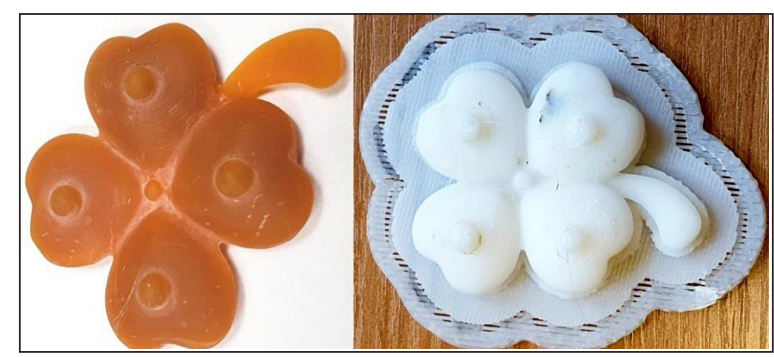

Fig. 12. Printed models with FDM and DPP technologies (from the left)

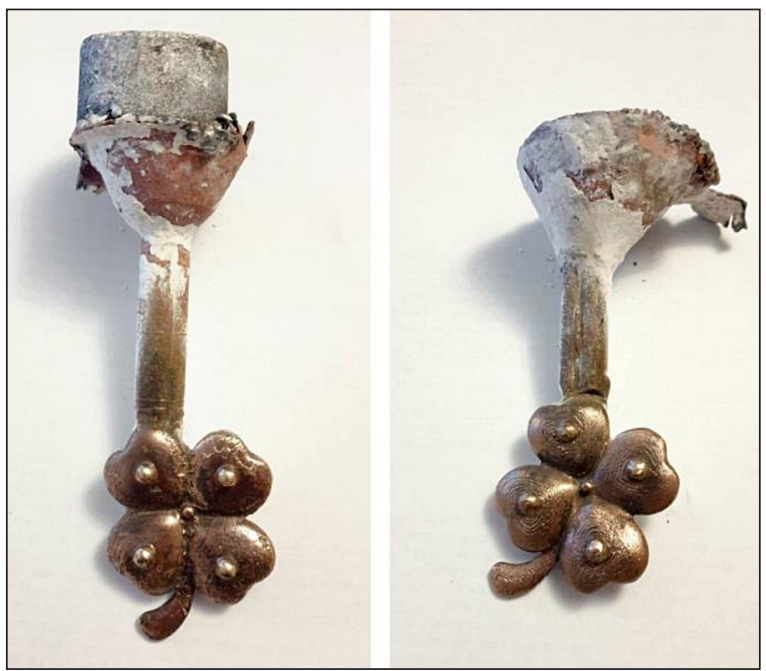

Fig. 13. Castings made from DPP and FDM models (from the left)

Table 1. The process data of 3D printing used in the studies

\begin{tabular}{|c|c|c|c|c|}
\hline Printer & Technology & Material & Layer thickness [mm] & Print time \\
\hline Zortrax M200 & FDM & HIPS & 0.09 & $1 \mathrm{~h} 35 \mathrm{~min}$ \\
\hline Liquid Crystal HR & DPP & Castable Resin & 0.05 & $2 \mathrm{~h} 45 \mathrm{~min}$ \\
\hline
\end{tabular}

Table 2. The roughness Ra measurement results obtained from surface tests

\begin{tabular}{|c|c|c|}
\hline Measurement number & $\begin{array}{l}\text { Casting made on the basis of the DPP } \\
\text { photopolymer resin model }[\mu \mathrm{m}]\end{array}$ & $\begin{array}{l}\text { Casting made on the basis of the HIPS } \\
\text { thermoplastic polymer model }[\mu \mathrm{m}]\end{array}$ \\
\hline 1. & 0.417 & 1.628 \\
\hline 2. & 0.384 & 2.233 \\
\hline 3. & 0.334 & 1.787 \\
\hline 4. & 0.581 & 1.899 \\
\hline 5. & 0.604 & 2.280 \\
\hline 6. & 0.824 & 1.512 \\
\hline 7. & 0.429 & 2.067 \\
\hline 8. & 0.873 & 1.642 \\
\hline 9. & 0.557 & 2.431 \\
\hline 10. & 0.538 & 1.977 \\
\hline
\end{tabular}


Table 3. The calculated standard deviation obtained from the surface tests

\begin{tabular}{|c|c|c|}
\hline Specification & $\begin{array}{l}\text { Casting made on the basis of the } \\
\text { DPP photopolymer resin model }[\mu \mathrm{m}]\end{array}$ & $\begin{array}{c}\text { Casting made on the basis of the HIPS } \\
\text { thermoplastic polymer model }[\mu \mathrm{m}]\end{array}$ \\
\hline Average & 0.554 & 1.946 \\
\hline Standard deviation & 0.179 & 0.308 \\
\hline Min. Ra value & 0.334 & 1.512 \\
\hline Max. Ra value & 0.873 & 2.431 \\
\hline Interval & 0.539 & 0.919 \\
\hline Result of the Ra value & $0.554 \pm 0.179$ & $1.946 \pm 0.308$ \\
\hline
\end{tabular}

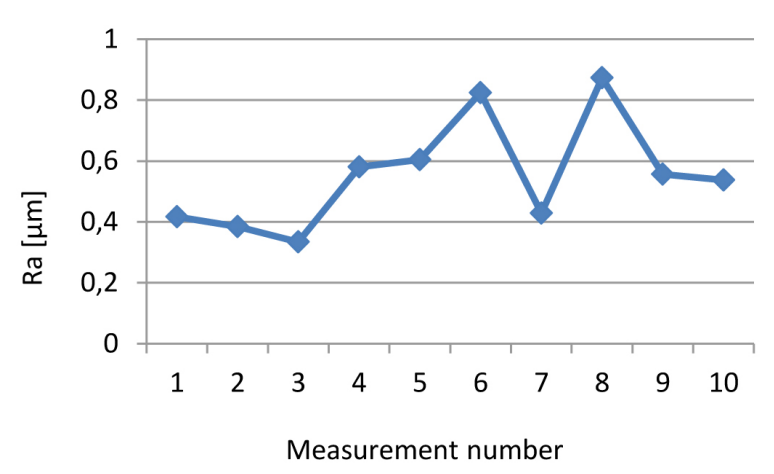

Fig. 14. Graph showing the value of the $\mathrm{Ra}$ index measured on a casting made on the basis of the resin model

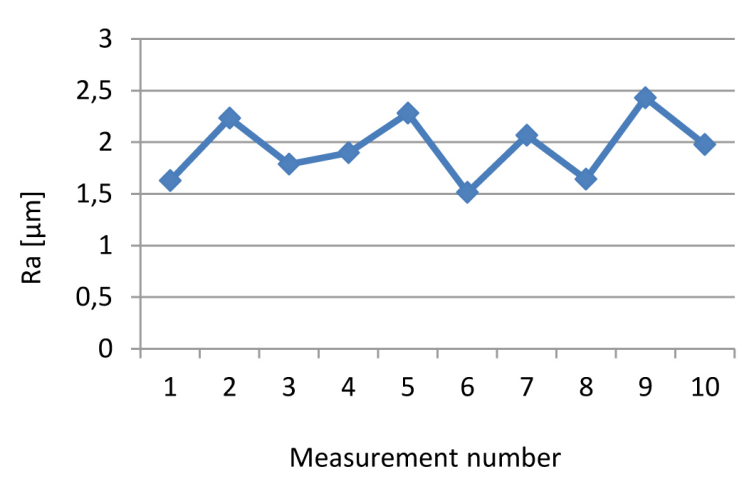

Fig. 15. Graph showing the value of the $\mathrm{Ra}$ index measured on the casting made on the basis of the HIPS model

\section{CONCLUSIONS}

In the article, the authors presented the process of obtaining and editing geometry on the example of an already existing jewelry. It was proven that the low-cost DAVID SLS-3 device is not suitable for digitizing precision products, in contrast to the Aicon SmartSCAN-HE R8. However, it should be emphasized that in both cases, the obtained model required an editing intervention by the operator. The last stage was the execution and measurement of the roughness of the castings made using the investment casting method which is considered to be one of the most accurate casting technologies. As a result of the measurements, it was shown that the use of DPP printing to make the casting models results in obtaining the castings with almost 3 times lower (max. value) roughness in relation to the FDM technology. Moreover, as a result of calculating the standard deviation, the deviation of the obtained roughness values should also be taken into account, which is halved for the castings based on the DPP technology, compared to those of FDM.

\section{REFERENCES}

1. Alic J., Computer-assisted everything? Tools and techniques for design and production, Technological Forecasting and Social Change, 44(4), 1993, 359-374, https://doi.org/10.1016/00401625(93)90041-5.

2. BaronioG., Volonghi P., Signoroni A., Concept and Design of a 3D Printed Support to Assist Hand Scanning for the Realization of Customized Orthosis, Applied Bionics and Biomechanics, 2017, Article ID 8171520, 8 pages. https://doi. org/10.1155/2017/8171520.

3. Bianco G., Gallo A., Bruno F., Muzzupappa M., A Comparative Analysis between Active and Passive Techniques for Underwater 3(13), 2013, $11007-$ 11031, doi:10.3390/s130811007.

4. Clarke T.A., Ellis T.J., Robson S., High accuracy 3-D measurement using multiple camera views, IEE Colloquium Digest No. 1994/054.

5. Cichalewski P., AutoCAD 3D - Model bryłowy i powierzchniowy, 2018, https://techtutor.pl/autocad-3d-2-model-brylowy-i-powierzchniowy/ (in Polish).

6. Daanen H.A.M, TerHaar F.B., 3D whole body scanners revisited, Displays, 34(4), 2013, 270-275, https://doi.org/10.1016/j.displa.2013.08.011. 
7. DREAM 3D, Photocentric Liquid Crystal HR, https:/www.dream3d.co.uk/product/photocentricliquid-crystal-hr/.

8. FORMLABS, Castable Wax: Jewelry Pattern Burnout Process, Retrieved 17.08.2020, https://media.formlabs.com/m/6b4018a61335afb9/original/ENUS-P-Castable-Wax-Jewelry-Pattern-BurnoutProcess.pdf.

9. Gorecky D., Schmitt, M., Loskyll M., Zuhlke D.: Human - machine - interaction in the Industry 4.0 ERA, Industrial Informatics (INDIN), 12th IEEE International Conference, 289-294 (2014).

10. Górski F., Wichniarek, R., Kuczko, W., Bun, P., Erkoyuncu, J.A.: Augmented Reality in Training of Fused Deposition Modelling Process, Advances in Manufacturing, Springer, 565-574 (2018).

11. Hajkowski J., Ignaszak Z., Experimental and simulation tests on the impact of the conditions of casting solidification from $\mathrm{AlSi} 9 \mathrm{Cu} 3$ alloy on their structure and mechanical properties, Archives of Foundry Engineering, 2018, 8(1), 167-175.

12. HEXAGON, SmartScan - The 3D optical scanner that's made to measure, Retrieved 17.08.2020, https://www.hexagonmi.com/en-US/products/ white-light-scanner-systems/aicon-smartscan.

13. Ignaszak Z., Popielarski P., Hajkowski J., Codina E., Methodology of comparative validation of selected foundry simulation codes, Archives of Foundry Engineering, 2015, 15(4), 37-44, DOI: 10.1515/AFE-2015-0076.

14. Ignaszak Z., Popielarski P., Sensitivity Tests of Simulation Models Used in Chosen Calculation Codes on Uncertainty of Thermo-Mechanical Parameters during Virtual Mechanical Stress Estimation for Ferrous Alloy Castings, Defect and Diffusion Forum, 2011, Vol. 312-315, 758-763, DOI: 10.4028/ WWW.SCIENTIFIC.NET/DDF.312-315.758.

15. Ignaszak Z., Sika R., Perzyk M., Kochański A., Kozłowski J., Effectiveness of SCADA systems in control of green sands properties, Archives of Foundry Engineering, 2016, 16(1), 5-12, DOI: 10.1515/afe-2015-0094.

16. Ignaszak Z., Sika R., Rogalewicz M., Contribution to the Assessment of the Data Acquisition Effectiveness in the Aspect of Gas Porosity Defects Prediction in Ductile Cast Iron Castings, Archives of Foundry Engineering, 2018, 18(1), 35-40, DOI: 10.24425/118808.

17. Ignaszak Z., Wojciechowski J., Analysis and Validation of Database in Computer Aided Design of Jewelery Casting, 2020, 2020(1), 9-16.

18. Jasiulewicz-Kaczmarek M., Legutko S., Kluk P., Maintenance 4.0 technologies - new opportunities for sustainability driven maintenance, Management and Production Engineering Review, 2020, 11(2), 74-87, DOI: 10.24425/mper.2020.13373.
19. Khan D., Shirazi M., Kim M.Y., Single shot laser speckle based 3D acquisition system for medical applications, Optical and Lasers in Engineering, 2018, 105, 43-53, https://doi.org/10.1016/j.optlaseng.2018.01.001

20. Kopecky M., Krejcovsky L., Svarc M., Antropometric Measuring Tools and Methodology for the Measurement of Anthropometric Parameters, Palacky University, Olomouc 2014.

21. Górski F., et al., 2019, Automated Design of Customized 3D-Printed Wrist Orthoses on the Basis of 3D Scanning. In: Computational and Experimental Simulations in Engineering : Proceedings of ICCES 2019, red. Hiroshi Okada, Satya N. Atluri: Springer International Publishing, 2020, 1133-1143.

22. Kujawinska, A., Vogt, K., Diering, M., Rogalewicz, M., Waigaonkar, S.D., Organization of visual inspection and its impact on the effectiveness of inspection, Lecture Notes in Mechanical Engineering, 2015, 19, 899-909, DOI: 10.1007/978-3-31968619-6_87.

23. Piperi E., Galantucci L. M., Kaçani J., Bodi I. and Spahiu T., From 3D scanning to 3D printing: Application in fashion industry, 7 th International Conference of Textile, 10-11 November, 2016, Tirana, Albania.

24. Raja V., Kiran J. F., Reverse Engineering, SpringerVerlag London, 2008.

25. Ramaraj T.C.,Eleftheriou E. C.,Ramaraj R., Integration of design and manufacture of complex geometries through solid and surface modeling techniques, Journal of Mechanical Working Technology, Vol. 20, September 1989, 141-152, DOI: 10.1016/0378-3804(89)90025-9.

26. Sepahvani P., Influences of additive manufacturing (3D printers) on the production cost and future of jewelry industry, Polytechnic University of Milan, Master of Science in Management Engineering, Department of Management, Economics and Industrial Engineering, 2016.

27. Sika R., Szajewski D., Hajkowski J., Popielarski P., Application of instance-based learning for cast iron casting defects prediction, Management and Production Engineering Review, 2019, 10(4), 101-107, DOI: $10.24425 /$ mper.2019.131450.

28. Spahiu T., Kacani J., Shehi E., Piperi E., 3D Body Scanning Technique for Anthropometric Measurements and Custom Clothing Designing, International Conference Developing Third Activities in Univesities, Albania 2014, DOI: 10.13140/2.1.1927.2484

29. Szelewski M., Wieczorowski M., Reverse Engineering and Methods of Discretization of Physical Objects, Stowarzyszenie Inżynierów I Techników Mechaników Polskich, 2015, 88(12), 183-188, DOI 10.17814/mechanik.2015.12.584. 
30. Żywicki, K., Zawadzki, P., Górski, F.: Virtual Reality Production Training System in the Scope of Intelligent Factory, Intelligent Systems in Production Engineering and Maintenance - ISPEM, Proceedings of the First International Conference on Intelligent Systems in Production Engineering and Maintenance ISPEM (2017).

31. Wannarumon S., Reviews of Computer-Aided Technologies for Jewelry Design and Casting, Department of Industrial Engineering, Naresuan University, 2011.

32. Wierzbicka N., et al., Prototyping of Individual Ankle Orthosis Using Additive Manufacturing Technologies Advances in Science and Technology Research Journal, 2017, 11(3), 283-288, DOI: $10.12913 / 22998624 / 76070$.

33. Vanderploeg A., The application of 3D printing technology in the fashion industry, 2017, 10(2), 170-179, DOI: 10.1080/17543266.2016.1223355.

34. Volonghi P., Signoroni A., Baronio G., 3D Scanning For Hand Orthotic Applications: A Comparative Assessment Between Static And Real-Time Solutions, in Proc. of 7th Int. Conf. on 3D Body
Scanning Technologies, Lugano, Switzerland, 2016, 61-69, DOI: 10.15221/16.061.

35. Yin Y., The evolution of production systems from Industry 2.0 through Industry 4.0, International Journal of Production Research, 2018, 56(1-2), 848-861, DOI: 10.1080/00207543.2017.1403664.

36. Zawadzki, P., Żywicki, K.,: Smart product design and production control for effective mass customization in the Industry 4.0 concept, Manag. and Prod. Eng. Rev., 2016, 7 (3), 105-112.

37. Zdobytskyi A., Lobur M., Iwaniec M., Breznitskyi V., Optimization of the Structural Characteristics of the Robotic System Holder, 15th International Conference on the Experience of Designing and Application of CAD Systems (CADSM), Polyana (Svalyava), Ukraine, IEEE, 2019.

38. Zhang, J., Yu, Z., Overview of 3D printing technologies for reverse engineering product design, Aut. Control Comp. Sc, 2016, 50, 91-97.

39. ZORTRAX Support Center, Printing tips, Retrieved 13.08.2020, https://support.zortrax.com/mseries-printing-tips/. 